\title{
The appropriateness of preoperative blood testing: A retrospective evaluation and cost analysis
}

\author{
H E Buley, ${ }^{1} \mathrm{MB}$ ChB; D Bishop, ${ }^{2} \mathrm{MB}$ ChB, FCA (SA); R Rodseth, ${ }^{2,3}$ MB ChB, FCA (SA), MMed, MSc, PhD \\ ${ }^{1}$ Department of Anaesthesiology and Critical Care, School of Clinical Medicine, College of Health Sciences, Nelson R Mandela School of \\ Medicine, University of KwaZulu-Natal, Durban, South Africa \\ ${ }^{2}$ Perioperative Research Group, Department of Anaesthetics and Critical Care, School of Clinical Medicine, College of Health Sciences, \\ Nelson R Mandela School of Medicine, University of KwaZulu-Natal, Durban, South Africa \\ ${ }^{3}$ Department of Outcomes Research, Cleveland Clinic, Cleveland, OH, USA
}

Corresponding author: H Buley (helenbuley@gmail.com)

Background. Inappropriate preoperative blood testing can negatively contribute to healthcare costs.

Objective. To determine the extent and cost implications of inappropriate preoperative blood testing in adult patients booked for orthopaedic, general or trauma surgical procedures at a regional hospital in KwaZulu-Natal Province, South Africa (SA).

Methods. We undertook a retrospective observational study using routine clinical data collected from eligible patient charts. The appropriateness of preoperative blood tests was evaluated against locally published guidelines on testing for elective and non-elective surgery. The cost of the relevant blood tests was determined using the National Health Laboratory Service 2014 State Pricing List. Results. A total of 320 eligible patient charts were reviewed over a 4-week period. Preoperative blood testing was performed in 318 patients. There was poor compliance with current departmental guidelines, with an estimated over-expenditure of ZAR81 019. Non-compliance was particularly prevalent in younger patients, patients graded as American Society of Anesthesiologists 1 and 2, and low-risk surgery groups. Conclusion. Inappropriate preoperative blood testing is common in our hospital, particularly in low-risk patients. This is associated with an increase in healthcare costs, and highlights the need for SA doctors to become more cost-conscious in their approach to blood testing practices.

S Afr Med J 2015;105(6):487-490. DOI:10.7196/SAMJ.9318

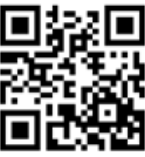

Preoperative evaluation, including blood testing, is an integral part of providing safe perioperative care. ${ }^{[1]}$ However, routine preoperative blood testing is no longer advocated, as several studies have highlighted the absence of negative outcomes associated with the omission of investigations and the low frequency of abnormal results in healthy patients undergoing low-risk surgery. ${ }^{[2,3]}$ Blood tests should therefore only be conducted when specifically indicated. In many centres, local guidelines have been developed to assist doctors in performing appropriate preoperative investigations.

In addition, inappropriate preoperative blood testing is costly. ${ }^{[4,5]}$ This is of particular importance in a highly cost-conscious environment such as the South African (SA) health sector. As stated by the National Treasury in 2013: ‘... departments and spending agencies do have to learn to do more with less. In the period ahead, improvements in outcomes have to come from qualitative improvements in the use of available budgets and other inputs. ${ }^{\text {'[6] }}$ The concept of 'costconsciousness' among doctors needs to become a more pertinent aspect of daily practice.

\section{Objective}

To determine the appropriateness of preoperative blood testing, and possible cost implications, in adult patients booked for orthopaedic, general or trauma surgical procedures at a regional hospital in KwaZulu-Natal Province, SA.

\section{Methods}

We conducted a retrospective observational study approved by the hospital manager, KwaZulu-Natal Department of Health, and the University of KwaZulu-Natal Biomedical Research Ethics Committee (Ref: BE345/14). A systematic convenience sampling method was used and data were collected retrospectively from eligible charts on an alternate week (Monday to Sunday) cycle over 8 weeks. This provided a total of 4 weeks of data collection over 2 consecutive months. Only routine clinical data from eligible charts were used. All adult patients ( $\geq 18$ years of age) undergoing elective or non-elective surgery in the disciplines of general, trauma or orthopaedic surgery were included. Patients requiring surgery while admitted to the intensive care, high-care and burn units were excluded, as were those who had undergone high-risk surgery within the last 6 months. Surgical risk was classified as low or high using previously published criteria. ${ }^{[7]}$

The primary study endpoint was the incidence of inappropriate preoperative blood tests across a range of laboratory tests. Those evaluated were full blood count (FBC), urea, creatinine and electrolytes (UE), liver function testing (LFT), the international normalised ratio (INR), blood gas analyses (ABG), calcium, magnesium and phosphate (CMP), C-reactive protein (CRP), erythrocyte sedimentation rate (ESR) and albumin. Results were expressed as a percentage of inappropriate tests against the total number of tests performed. The appropriateness of a blood test was determined by the Pietermaritzburg Metropolitan Department of Anaesthesia's guidelines on preoperative blood testing for elective and non-elective surgery (Appendix 1). Where the appropriateness of preoperative blood testing could not be ascertained clearly, the relevant data were reviewed separately by $\mathrm{DB}$ and $\mathrm{RR}$, both anaesthetic consultants familiar with the departmental protocol.

The cost implication of unnecessary preoperative blood testing was determined by multiplying the cost of the relevant blood test by the number of unnecessary respective tests performed in the sample. The cost of the relevant blood tests was obtained from the National Health Laboratory Service (NHLS) 2014 State Pricing List. 


\section{Results}

\section{Cohort characteristics}

A total of 320 eligible patient charts were reviewed, and the characteristics of the cohort are summarised in Table 1. The majority of surgical procedures at the study hospital are done on low-risk patients (81.9\%), patients who are $\leq 60$ years of age $(84.4 \%)$ and those who have an American Society of Anesthesiologists (ASA) physical status grading of 1 or 2 (86.9\%). Comorbidities observed included asthma, diabetes, vascular disease, hypertension, epilepsy and HIV infection. The charts reflected 131 (40.9\%) general surgical, 15 (4.7\%) trauma and 174 (54.4\%) orthopaedic surgery cases.

\section{Preoperative testing practices}

New preoperative blood testing was performed either on admission or before surgery on all but two of the 320 patients evaluated. Only one patient had no preoperative testing performed, and one patient had results from the referral hospital with no subsequent testing. Eight of the included patients had more than one surgical procedure, with no repeat testing between procedures. The most commonly performed preoperative blood tests were an FBC (310) and UE (310). Additional frequently performed tests included LFT, INR, ABG, CMP, CRP, ESR and albumin. Observed preoperative testing practices are summarised in Table 2.

Inappropriate tests included $190 \mathrm{FBCs}$ (61.3\%), 206 UEs (66.4\%), 92 LFTs (97.9\%) and 49 INRs $(92.5 \%)$. The majority of patients who received inappropriate tests were $<45$ years of age, graded as ASA 1 , or undergoing a low-risk surgical procedure. In addition, we noted that four patients were discharged after surgery without their blood results being documented in their files. Further, in 60 charts the FBC and
UE were repeated perioperatively without any clear indication, and in seven charts admission blood tests were performed twice within 24 hours. With no clearly delineated surgical, trauma or orthopaedic preoperative guidelines, it was not possible to determine the appropriateness of blood testing for surgical purposes relative to anaesthetic indications.

The documentation of blood results on anaesthetic forms was also reviewed. No blood results were documented on $11.9 \%$ of charts, $24.0 \%$ of charts had documented ward haemoglobin only, and $1.6 \%$ had a blood gas result only. Adequate documentation for appropriate FBCs and UEs was observed in $70.0 \%$ and $67.3 \%$ of charts, respectively. Despite the high prevalence of unnecessary blood testing, there was also inadequate documentation of appropriate tests on the anaesthetic charts.

\section{Cost implications}

The total cost of the inappropriate tests performed was ZAR72 375 . The largest contributors to this included 92 LFTs (ZAR29 349), 206 UEs (ZAR15 817) and the combined group of CMP, albumin, ESR and CRP (ZAR12 566). The cost of repeat and duplicate testing practices that were observed was ZAR8 643. In total, the estimated cost of unnecessary perioperative testing in a 4-week period was ZAR81 018

\section{Discussion}

This study demonstrates that inappropriate blood testing is common in patients booked for both elective and non-elective orthopaedic, trauma or general surgical procedures - this despite the existence of locally developed anaesthetic preoperative testing guidelines. The majority of procedures that take place at the study hospital involve low-risk surgery in patients
$<60$ years of age and with an ASA grading of 1 or 2 . These patient groups undergo unnecessary testing that has significant cost implications.

The role of preoperative blood testing is to assist in the detection of abnormalities that could alter patient management and lead to better outcomes. In a recent study, Benarroch-Gampel et al. ${ }^{[3]}$ examined patterns of preoperative blood testing in 73596 patients undergoing elective hernia repair, of whom 46977 underwent testing. Tests included FBC, creatinine, electrolytes, LFT and coagulation parameters. Their findings suggested that a large proportion of testing for low-risk ambulatory surgery, even in patients with stable comorbid illness, is of questionable clinical benefit and can

Table 1. Cohort characteristics of the charts reviewed $(N=320)$

\begin{tabular}{ll}
\hline & $\boldsymbol{n}(\%)$ \\
\hline Surgical risk & \\
Low risk & $262(81.9)$ \\
$\quad$ High risk & $58(18.1)$ \\
Surgical urgency & \\
$\quad$ Non-elective & $178(55.6)$ \\
Elective & $142(44.4)$ \\
Age group (years) & \\
$<45$ & $203(63.4)$ \\
$45-60$ & $67(20.9)$ \\
$61-75$ & $42(13.1)$ \\
$>75$ & $8(2.5)$ \\
ASA grading & \\
1 & $137(42.8)$ \\
2 & $141(44.1)$ \\
3 & $39(12.2)$ \\
4 & $3(0.9)$
\end{tabular}

Table 2. Observed preoperative testing practices

\begin{tabular}{|c|c|c|c|c|c|c|c|c|}
\hline \multirow[b]{2}{*}{ Blood test } & \multirow{2}{*}{$\begin{array}{l}\text { Total } \\
N\end{array}$} & \multirow{2}{*}{$\begin{array}{l}\text { Surgical risk low } \\
n(\%)\end{array}$} & \multicolumn{3}{|c|}{ Age group (years) $n(\%)$} & \multicolumn{3}{|c|}{ ASA grading $n(\%)$} \\
\hline & & & $<45$ & $45-60$ & $>61$ & 1 and 2 & 3 & 4 \\
\hline FBC & 310 & $253(81.6)$ & $195(62.9)$ & $67(21.6)$ & $48(15.5)$ & $270(87.1)$ & $37(11.9)$ & $3(1.0)$ \\
\hline UE & 310 & $253(81.6)$ & $194(62.9)$ & $67(21.6)$ & 49 (15.8) & $269(86.8)$ & $38(12.3)$ & $3(1.0)$ \\
\hline LFT & 94 & $65(69.1)$ & $54(57.4)$ & $21(22.3)$ & $19(20.2)$ & $75(79.8)$ & $17(18.1)$ & $2(2.1)$ \\
\hline CMP & 81 & $65(80.2)$ & $41(50.6)$ & $20(24.7)$ & $20(24.7)$ & $62(76.5)$ & $18(22.2)$ & $1(1.2)$ \\
\hline CRP & 55 & $44(80.0)$ & $38(69.1)$ & $10(18.2)$ & $7(12.7)$ & $49(89.1)$ & $6(10.9)$ & - \\
\hline $\mathrm{ABG}$ & 54 & $35(64.8)$ & $33(61.1)$ & $14(25.9)$ & $7(13.0)$ & $43(79.6)$ & $9(16.7)$ & $2(3.7)$ \\
\hline INR & 53 & $43(81.1)$ & $27(50.9)$ & $15(28.3)$ & $11(20.8)$ & $39(73.6)$ & $12(22.6)$ & $2(3.8)$ \\
\hline ESR & 41 & $32(78.0)$ & $29(70.7)$ & 7 (17.1) & $5(12.2)$ & $38(92.7)$ & $3(7.3)$ & - \\
\hline Albumin & 35 & $28(80.0)$ & 15 (42.9) & $12(34.3)$ & $8(22.9)$ & $28(80.0)$ & $6(17.1)$ & $1(2.9)$ \\
\hline
\end{tabular}


be eliminated without significant adverse medical consequences.

Surgical indications for specific tests must also be addressed, e.g. LFTs, albumin, ESR, CRP and CMP. Their place in perioperative care needs to be challenged, especially in young, healthy patients undergoing low-risk surgery.

Lilford et al. ${ }^{[8]}$ reviewed a cohort of 1290 patients with abnormal LFT results. They concluded that LFT was associated with a high false-positive rate and often performed for reasons other than that of clinical indication. They advocate a more selective approach to LFT instead of screening all liver enzymes.

Albumin is a controversial biomarker of nutritional status, as many other factors, including inflammation, metabolic stress, trauma and dehydration, can affect serum levels. ${ }^{[9]}$ Studies suggest that it can be used as a prognostic marker to identify those at risk of complications following surgery and poor postoperative outcome. ${ }^{[9]}$ However, this is not applicable in patients undergoing lowrisk surgery.

Both the ESR and CRP are nonspecific tests that reflect inflammation associated with infection or autoimmune disease. CRP in particular can be used as a monitor for postoperative infection and could assist in identifying patients who are developing severe sepsis. ${ }^{[10]}$ However, once again their indication in the healthy patient undergoing low-risk surgery must be questioned.

Disorders in mineral metabolism are complex. Multiple organ systems, in particular the bone mineral content, neurological and cardiovascular systems, can be affected by altered levels of calcium, magnesium and phosphate. ${ }^{[11]}$ Critical illness can have various effects on CMP levels, for multiple reasons. This would justify regular CMP monitoring in critical illness. However, CMP testing in the majority of patients is of questionable value without clear clinical indications.

Numerous international studies have highlighted the cost implications of inappropriate blood testing. ${ }^{[4,5]}$ It must also be emphasised that testing not only has financial implications but means unnecessary discomfort to patients, increased occupational exposure to the risk of needlestick injuries, and time lost through acquiring blood samples, waiting for results and addressing false-positive tests.

Perioperative care should be a multidisciplinary process involving both the surgeon and the anaesthetist. Addressing unnecessary preoperative blood testing practices should not be the sole responsibility of the surgeon or the anaesthetist, but rather a process of information sharing and resource management. This study highlights that inappropriate preoperative blood testing is evident despite available anaesthetic guidelines.

Reasons for non-compliance were not explored in this study. However, a qualitative study by Brown and Brown ${ }^{[12]}$ identified various factors responsible for unnecessary testing despite available guidelines. These included: (i) lack of awareness of guidelines; (ii) medicolegal concerns; (iii) concern about surgical delays or cancellations; and (iv) the belief that other physicians may require the test results. Surgeons and anaesthetists need to collaborate to ensure knowledge translation and together address implementation issues around current preoperative testing guidelines.

Administrative interventions must also be instituted. ${ }^{[13]}$ The NHLS has implemented some of these through electronic gatekeeping, limiting volumes and type of tests ordered, but this tool is not yet available at all state hospitals. ${ }^{[14]}$ Further examples of such interventions include: (i) modification of laboratory request forms to limit available options; (ii) imposing a specific time interval on subsequent testing to prevent repeat testing; (iii) restricting the ordering of specific tests to consultant request only; and (iv) encouraging a selective testing approach, as summarised in Table 3.

Finally, the current literature on preoperative testing is based on US,
Canadian or European population groups. The SA population has lower socioeconomic living conditions than those in the northern hemisphere, and disease profiles differ. There is no literature validating preoperative testing guidelines appropriate to the SA population at present, and future studies are required. Reasons behind local preoperative blood testing practices and non-compliance with guidelines also need to be explored and addressed.

\section{Study limitations}

Given that this was a retrospective study, all study data were limited by the subjectivity of the attending doctor's assessment of the clinical condition of the patient and the ASA grading. Furthermore, at the time of the study, no clearly delineated surgical or orthopaedic guidelines were available regarding preoperative investigations required.

\section{Conclusion}

This study demonstrates that inappropriate blood testing often occurs in patients booked for elective and non-elective general, orthopaedic and trauma surgical procedures, and that it was common in the younger patient, ASA 1 or 2 and low-risk surgery groups. Mis- and overutilisation of blood testing has a significant impact on healthcare costs. SA doctors need to become more cost-conscious in their approach to laboratory testing practices in preoperative patients.

\section{Table 3. Options available for a selective testing approach}

\begin{tabular}{lll}
\hline Test required & Test options & Cost implications \\
\hline $\mathrm{Hb}$ & FBC & ZAR52.23 \\
& FBC and differential & ZAR80.87 (ZAR52.23 + ZAR28.64) \\
& Hb only & ZAR16.24 \\
& Hb POCT ${ }^{*}$ & Unknown: cost-effective ${ }^{[15]}$ \\
Albumin & Albumin only & ZAR36.23 \\
& Albumin as part of an LFT & ZAR319.01 \\
LFT & LFT & ZAR319.01 \\
& Selective test & \\
& Bilirubin total & ZAR31.77 \\
& Liver enzyme & ZAR40.91 \\
& Protein total & ZAR23.48 \\
& CMP & ZAR81.96 \\
CMP & Selective test & \\
& Calcium only & ZAR27.32 \\
& Magnesium only & ZAR27.32 \\
& Phosphate only & ZAR27.32 \\
&
\end{tabular}




\section{References}

1. Task Force on Preanesthesia Evaluation. Practice advisory for preanesthesia evaluation: A report by the American Society of Anesthesiologists Task Force on preanesthesia evaluation. Anesthesiology 2002;96(2):485-496. [http://dx.doi.org/10.1097/00000542-200202000-00037]

2. Kumar A, Srivastava U. Role of routine investigations in preoperative evaluation. J Anaesthesiol Clin Pharmacol 2011;27(2):174-179. [http://dx.doi.org/10.4103/0970-9185.81824]

3. Benarroch-Gampel J, Sheffield KM, Duncan CB, et al. Preoperative laboratory testing in patients undergoing elective, low-risk ambulatory surgery. Ann Surg 2012;256(3):518-528. [http://dx.dol. org/10.1097/SLA.0b013e318256bcdb]

4. Mancuso CA. Impact of new guidelines on physicians' ordering of preoperative tests. J Gen Intern Med 1999;14(3):166-172. [http://dx.doi.org/10.1046/j.1525-1497.1999.00308.x]

5. Siriussawakul A, Nimmannit A, Rattana-arpa S, et al. Evaluating compliance with international Siriussawakul A, Nimmannit A, Rattana-arpa S, et al. Evaluating compliance with international
preoperative testing guidelines for minimal-risk patients undergoing elective surgery. Biomed Res Int 2013;2013: Article ID 835426. [http;//dx.doi.org/10.1155/2013/835426]

6. Fuzile L. Estimates of National Expenditure 2013. Pretoria: National Treasury, Republic of South Africa, 2013:5. http://www.treasury.gov.za/documents/national\%20budget/2013 (accessed 11 May 2015).

7. Devereau PJ, Chan MTV, Alonso-Coello P, et al. Association between postoperative troponin levels and 30 day mortality among patients undergoing noncardiac surgery. JAMA 2012;307(21):2295-2304. [http://dx.doi.org/10.1001/jama.2012.5502]
8. Lilford RJ, Bentham L, Girling A, et al. Birmingham and Lambeth liver evaluation testing strategies (BALLETS) A prospective cohort study. Health Technol Assess 2013;17(28):i-xiv,1-307. [http://dx.doi.org/10.3310/hta17280] 9. Basu I, Subramanian P, Prime M, et al. The use of biochemical parameters as nutritional screenin tools in surgical patients. Surgical Science 2011;2(2):89-94. [http://dx.doi.org/10.4236/ss.2011.22019] 10. Faix JD. Biomarkers of sepsis. Crit Rev Clin Lab Sci 2013;50(1):23-36. [http://dx.doi.org/10.3109/10 408363.2013.764490]

11. Baker SB, Worthley LIG. The essentials of calcium, magnesium and phosphate metabolism: Part II. Disorders. Crit Care Resusc 2002;4(4):307-315. [PMID: 16573444]

12. Brown SR, Brown J. Why do physicians order unnecessary preoperative tests? A qualitative study. Fam Med 2011;43(5):338-343.

13. Stuebing EA, Milner TJ. Surgical vampires and rising health care expenditure. Arch Surg 2011;146(5):524-527. [http://dx.doi.org/10.1001/archsurg.2011.103]

14. Pillay S. National Health Laboratory Service Annual Report 2013-2014. Johannesburg. National Health Laboratory Service, 2014:12.
Heatlay S. National Health Laboratory

15. Mimoz O, Frasca D, Médard A, et al. Reliability of the HemoCue ${ }^{\Theta}$ hemoglobinometer in critically ill patients: A prospective observational study. Minerva Anestesiol 2011;77(10):979-985.

Accepted 21 April 2015 


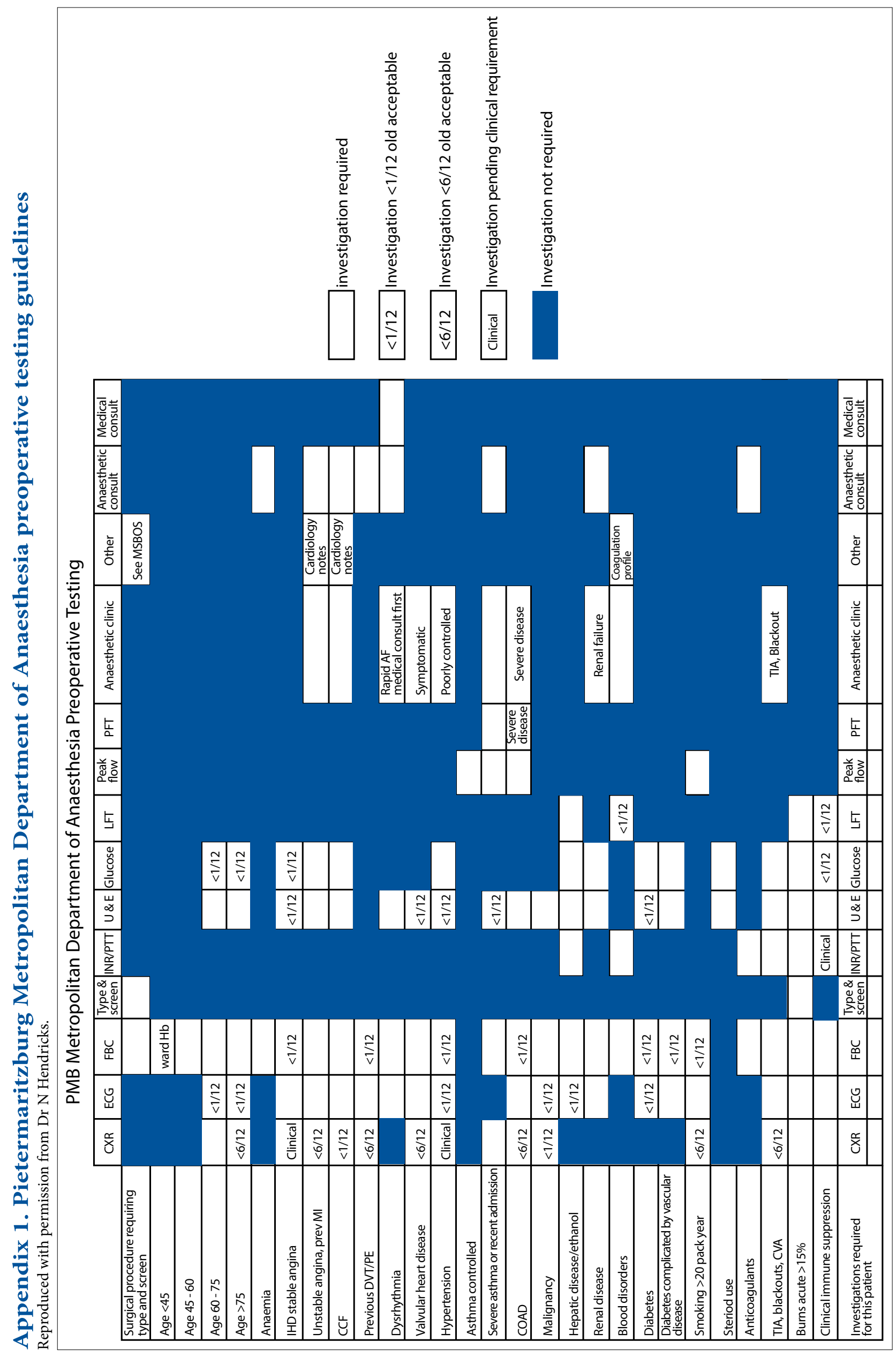

